Revue internationale P.M.E.

Économie et gestion de la petite et moyenne entreprise

Revue

internationale

PME

\title{
Stratégies de croissance et contexte de gouvernement des entreprises de biotechnologies
}

\section{Jean-Pierre Boissin et Michel Trommetter}

Volume 16, numéro 3-4, 2003

URI : https://id.erudit.org/iderudit/1008446ar

DOI : https://doi.org/10.7202/1008446ar

Aller au sommaire du numéro

Éditeur(s)

Presses de l’Université du Québec

ISSN

0776-5436 (imprimé)

1918-9699 (numérique)

Découvrir la revue

Citer cette note

Boissin, J.-P. \& Trommetter, M. (2003). Stratégies de croissance et contexte de gouvernement des entreprises de biotechnologies. Revue internationale P.M.E., 16(3-4), 75-93. https://doi.org/10.7202/1008446ar

\section{Résumé de l'article}

L'industrie des biotechnologies peut être représentée par différentes activités et différentes stratégies de croissance de l'entreprise. Ces stratégies auront un impact sur la structure de propriété et la qualité des apporteurs de capitaux à mobiliser, donc sur les mécanismes de gouvernement d'entreprise à mettre en place. On passe d'un système de gouvernement lié à des entreprises entrepreneuriales, avec des informations classiques, à un système de gouvernement dynamique (anticipation de la séquentialité des différentes phases de la recherche) dans lequel la firme est dépendante des financements, à chaque étape de sa croissance. Les informations qualitatives, notamment en matière de R-D, sont alors déterminantes. Les nombreuses entreprises de biotechnologies, sans chiffre d'affaires et avec moins de 50 salariés, relèvent de la moyenne entreprise au regard d'un processus de séparation des droits de contrôle et de gestion et d'une ouverture du capital favorisant la transférabilité des droits. 


\title{
Note de recherche \\ Stratégies de croissance et contexte de gouvernement des entreprises de biotechnologies ${ }^{1}$
}

\author{
Jean-Pierre BOISSIN \\ Michel TROMMETTER \\ Université Pierre-Mendès-France
}

\section{MOTS CLÉS}

\section{Gouvernement d'entreprise - Entreprises de biotechnologies Stratégies de croissance - Structures de propriété Moyenne entreprise}

\begin{abstract}
LES AUTEURS
JEAN-PIERRE BoISSIN est enseignant-chercheur en sciences de gestion au CERAG à ll'Université Pierre-Mendès-France (Grenoble, France). Ses travaux de recherche portent sur les stratégies de croissance et le gouvernement d'entreprise, l'entrepreneuriat et la structuration du management stratégique. Il est responsable de la Maison de l'entrepreneuriat interuniversitaire de Grenoble et Secrétaire général de l'association Vouloir Entreprendre (réseau pour la sensibilisation pédagogique à l'entrepreneuriat). Adresse : CERAG, Université Pierre-Mendès-France, B.P. 47, 38040 Grenoble Cédex 9, France. Tél. : 3304768254 14. Courriel : <jean-pierre. boissin@upmf-grenoble.fr>.

Michel TROMmetTer est chercheur en sciences économiques dans l'unité de recherche GAEL de l'INRA et de l'Université Pierre-Mendès-France (Grenoble, France). Ses travaux de recherches portent sur l'approche séquentielle de la décision en incertitude et sur la définition des droits de propriété intellectuelle dans les biotechnologies. II est membre de la commission scientifique de l'Institut français de la biodiversité (IFB). Adresse : INRA / GAEL, Université Pierre-Mendès-France, B.P. 47, 38040 Grenoble Cédex 9, France. Tél. : 330476825414. Courriel : <michel.trommetter@grenoble.inra.fr>.
\end{abstract}

1. Les résultats de cet article sont basés sur une recherche financée par le Commissariat général du Plan français. Nous remercions les dirigeants d'entreprise qui ont accepté de nous rencontrer lors de cette recherche. Les erreurs éventuelles de ce papier restent de notre entière responsabilité. 


\title{
RÉSUMÉ
}

L'industrie des biotechnologies peut être représentée par différentes activités et différentes stratégies de croissance de l'entreprise. Ces stratégies auront un impact sur la structure de propriété et la qualité des apporteurs de capitaux à mobiliser, donc sur les mécanismes de gouvernement d'entreprise à mettre en place. On passe d'un système de gouvernement lié à des entreprises entrepreneuriales, avec des informations classiques, à un système de gouvernement dynamique (anticipation de la séquentialité des différentes phases de la recherche) dans lequel la firme est dépendante des financements, à chaque étape de sa croissance. Les informations qualitatives, notamment en matière de $R$ - $D$, sont alors déterminantes. Les nombreuses entreprises de biotechnologies, sans chiffre d'affaires et avec moins de 50 salariés, relèvent de la moyenne entreprise au regard d'un processus de séparation des droits de contrôle et de gestion et d'une ouverture du capital favorisant la transférabilité des droits.

\begin{abstract}
Different activities and different growth strategies of firm can represent the biotechnology industry. These strategies will have an impact on the property structure and the quality of the capital suppliers, therefore on the government mechanisms to set up firms. One goes from governance linked to entrepreneurial firms, with classical informations, to a dynamic governance system (anticipation of the sequentiality of the different phases of the research) in which the firm is dependent on the financings at every step. Quality informations, notably regarding to the research development, are then determining. The numerous biotechnology firms, without turnover and with less than fifty employees, rise from the small and medium enterprise with a separation process of the control rights and of management, and opening the capital favoring the right transferability.
\end{abstract}

\section{RESUMEN}

La industria biotecnológica puede ser representada por varias actividades y varias estrategias de crecimiento de la empresa. Estas estrategias van a tener consecuencias sobre la estructura de la propiedad y la calidad de los que van a traer capitales a mobilizar, y pues sobre los mecanismos de gobierno de la empresa que se deben organizar. Pasamos de un sistema de gobierno ligado a empresas emprendedoras, con informaciones clásicas, a un sistema de gobierno dinámico (con anticipacion de la secuencialidad de las diferentes etapas de la investigación) en el cual la empresa es dependente de las financiaciones, a cada etapa. Las informaciones cualitativas, especialmente tratando de investigación y desarrollo, son determinantes. Las numerosas empresas de biotecnológica, sin volumen de negocios, con menos de cincuenta asalariados, pertenecen al grupo de las empresas medianas, por consecuencia de un proceso de separación de los derechos de control y de gestión y de una apertura del capital que favorece la transferabilidad de los derechos. 


\section{ZUSAMMENFASSUNG}

Die Biotechnologieindustrie kann durch verschiedene Aktivitäten und unterschiedliche Wachstumsstrategien von Unternehmen dargestellt werden. Diese Strategien haben Auswirkungen auf die Eigentumsstruktur und die Qualität, Kapitalgeber zu mobilisieren. Folglich sind auch Auswirkungen auf die zu realisierenden Mechanismen der Unternehmensführung und Unternehmensaufsicht vorhanden. Es kann der Übergang festgestellt werden von einem unternehmerzentrierten System der Unternehmungsführung mit klassischen Informationsmodellen, zu einem dynamischen Führungssystem (Mit der Vorwegnahme der verschiedenen Phasen der Forschung). In diesem dynamischen System ist die Unternehmung in jeder Phase ihres Wachstums von der Finanzierung abhängig. Die qualitativen Informationen, vor allem hinsichtlich der Entwicklungsuntersuchung sind in der Folge entscheidend. Die zahlreichen Biotechnologieunternehmen ohne Umsatz und mit weniger als 50 Mitarbeitern, haben eine Sonderstellung im Hinblick auf die Trennung zwischen dem Kontrollanspruch und der Verwaltung sowie hinsichtlich der Öffnung des Kapitals. Die Öffnung des Kapitals bevorteilt die Übertragbarkeit der Anspruchsrechte.

\section{Introduction}

Dans le cadre des analyses sur la nouvelle économie, deux secteurs sont particulièrement cités : les technologies de l'information et les biotechnologies. De nombreux travaux sur les entreprises de biotechnologies ont porté, soit sur leur organisation et principalement sur la relation entre la localisation d'une firme et ses réseaux de collaborations scientifiques (Audrescht et Stephan, 1996), soit sur la relation entre entreprises privées et laboratoires publics de recherches (Joly, Lemarié et Mangematin, 1998), soit sur l'importance de la dynamique industrielle dans les biotechnologies (Mignot et Poncet, 2001).

Dans ces différents travaux, les entreprises sont censées appartenir à un secteur industriel homogène à forte croissance, celui des biotechnologies. Or, il apparaît possible de distinguer différentes activités selon les variables de segmentation choisies tels que les marchés aval (pharmacie, agroalimentaire, etc.) ou surtout les processus innovants mis en œuvre (service, produits, taille des programmes de recherche-développement). Ainsi, il ressort que toutes les entreprises de biotechnologies ne sont pas des entreprises de haute technologie à forte potentialité de croissance. Les objectifs de croissance sont variables (Mangematin et al., 2003). C'est pourquoi dans la continuité de ces derniers résultats, nous allons étudier les stratégies de croissance et leurs implications sur le contexte du gouvernement de 60 entreprises de biotechnologies en France, à partir d'entretiens avec leurs dirigeants. Trois principales dimensions ont été retenues : le profil du dirigeant, les stratégies de croissance et les structures de propriété. 
La méthodologie d'enquête est présentée dans une première section. Ensuite, les liens entre les stratégies de croissance, les profils de management des dirigeants et les contraintes de financement sont analysés dans une deuxième section. Enfin, dans une troisième section, le lien entre stratégies de croissance et structures de propriété est établi. Au final, une contextualisation des mécanismes de gouvernement d'entreprise dans l'industrie des biotechnologies est proposée.

\section{Méthodologie d'enquête sur les entreprises françaises de biotechnologies ${ }^{2}$}

L'origine de la base de données et les grandes variables retenues seront tour à tour examinées.

\subsection{Construction de la base de données}

Les informations ont été recueillies entre janvier et juin 2000 par des chercheurs en binôme à partir d'entretiens semi-directifs auprès de 60 dirigeants d'entreprises françaises de biotechnologies figurant dans la base de données du ministère français de la Recherche et des Technologies. Cette méthode de collecte de données a l'avantage de déboucher sur un échantillon représentatif et de procéder par études de cas ouvertes à des informations non anticipées (voir la méthodologie proche utilisée récemment par Julien [2001] et les recommandations de Huberman [1981] et Huberman et Miles [1991]).

L'enjeu reposait sur l'accès à des données relevant davantage du discours du dirigeant sur son environnement et en particulier, avec ses partenaires pour apprécier les contextes du gouvernement de l'entreprise. L'intérêt des discours des acteurs de l'action stratégique a déjà été souligné comme moyen pour comprendre les représentations véhiculées (Martinet, 1990, 1992 ; Mounoud, 1997). Une partie des données pouvait faire l'objet d'une triangulation avec des informations secondaires (site Internet de l'entreprise, base de données financières, presse spécialisée, rapports annuels).

La conduite de l'entretien s'est articulée à partir de six grandes parties : l'identité du dirigeant et la structuration des ressources humaines, l'historique de la stratégie de croissance de l'entreprise depuis sa création, la description du positionnement concurrentiel avec la caractérisation de l'activité (technologie, brevets déposés, situation dans la filière, phase de développement), les relations et les alliances avec les différents partenaires (clients type grande entreprise pharmaceu-

2. Présentation inspirée de la grille d'analyse de structuration d'un article proposée par Castagnos, Boissin et Guieu (1997). 
tique ou agrochimique ou cosmétique ; fournisseurs type laboratoire de recherche public ou privé ; rôle des pouvoirs publics ; etc.), le descriptif et le rôle des actionnaires et administrateurs, la place des acteurs publics (Anvar, ministères, collectivités territoriales).

Les résultats présentés ici ne couvrent qu'une partie des données.

\subsection{Présentation des données utilisées}

Deux types de données ont été exploités :

- des données quantitatives ayant trait essentiellement à la comptabilité financière : chiffre d'affaires (CA), résultats d'exploitation (RE), résultat net $(\mathrm{RN})$, fonds propres (FP), capital social (KS), dettes, effectif, nombre de brevets, date de création.

- des données qualitatives par une codification des entretiens: profil du dirigeant (qualité de la formation initiale, fondateur ou non); présence d'un comité scientifique (CS) et notoriété de sa composition (Prix Nobel), l'aide de l'Anvar, l'existence de salariés actionnaires ou non.

Deux variables qualitatives ont été construites pour mettre en relief les contextes de gouvernement d'entreprise. En effet, l'activité comme élément principal de la stratégie de croissance et la structure de propriété de l'entreprise doivent offrir des éléments de contextualisation des mécanismes de gouvernement d'entreprise.

Le concept d'activité recouvre une diversité de réalité de terrain :

- les entreprises de type 1 offrent de nouveaux produits par création de marchés de niche;

- les entreprises de type 2 proposent des prestations de services aussi sur des marchés de taille limitée;

- les entreprises dites de type 3 vendent des prestations de service à de grands groupes industriels, auxquels elles consentent des contrats d'exclusivité ;

- les entreprises dites de type 4 assurent elles-mêmes d'importants programmes de recherche (mise au point d'une nouvelle molécule) sur des marchés aval de grande taille avec de fortes perspectives de croissance. Suivant le stade d'avancement du projet, deux sous-types sont distingués :

- la PME ne réalise pas encore de ventes et entre dans le type 4a;

- ensuite, une fois que la mise sur le marché est proche, la PME entre dans le type $4 b$. 
Chaque entreprise appartient d'abord à l'une de ces activités bien qu'elle puisse parfois être dans une phase de transition entre deux activités.

Les entreprises ont aussi été caractérisées par leur structure de propriété à travers la qualité des actionnaires :

Catégorie 1. Les entreprises indépendantes à actionnaires privés (individus), parfois ouvertes sur des fonds d'investissements régionaux (collectivités territoriales), dites entrepreneuriales.

Catégorie 2. Les filiales de groupes pharmaceutiques ou agroalimentaires ou cosmétiques ou de holdings financiers de groupes de biotechnologies.

Catégorie 3. Les entreprises avec des investisseurs de capital-risque (autres que régionaux) selon les minorités de blocage potentiel dans les conseils d'administration ou de surveillance :

- 3a: capital-risque $<30 \%$ du capital ;

- 3b : capital-risque 30-49\% du capital ;

- 3c : capital-risque $+50 \%$ du capital.

Catégorie 4. Les entreprises à actionnaires «publics» au sens anglo-saxon, cotées sur les marchés financiers.

\section{Stratégies de croissance, profils des dirigeants et contrainte de financement}

L'analyse s'appuie sur un modèle simple. La stratégie de croissance relève du dirigeant et, donc, de son profil. Cette stratégie de croissance se traduit par des contraintes de financement distinctes.

\subsection{Entrepreneurs et managers des entreprises de biotechnologies}

La représentation scolastique du profil du dirigeant de l'entreprise, à savoir l'entrepreneur versus le manager, est-elle opérante dans l'échantillon ${ }^{3}$ ? Ces deux profils peuvent-ils être rapprochés des différentes stratégies de croissance ? C'est ce que nous allons voir à partir du tableau 1 .

3. Les tests de chi carré sont significatifs, mais une partie des effectifs théoriques est inférieure à 5 , ce qui limite la portée des résultats. 


\section{TABLEAU 1}

Stratégies de croissance et variables de management du dirigeant

\begin{tabular}{lrrccccr}
\hline $\begin{array}{l}\text { Stratégies de croissance } \\
\text { Variables } \\
\text { de management }\end{array}$ & $\begin{array}{c}\mathbf{1} \\
\text { Niche } \\
\text { produit }\end{array}$ & $\begin{array}{c}\text { Niche } \\
\text { service }\end{array}$ & $\begin{array}{c}\text { Service } \\
\text { par contrat } \\
\text { recherche }\end{array}$ & $\begin{array}{c}\text { Ra } \\
\text { R-D }\end{array}$ & $\begin{array}{c}\text { 4b } \\
\text { Entrée } \\
\text { marché }\end{array}$ & Total & 4a+b \\
\hline Total & 18 & 19 & 6 & 9 & 8 & 60 & 17 \\
\hline Scientifique & 13 & 15 & 4 & 6 & 4 & $42 / 60$ & 10 \\
Gestionnaire & 5 & 4 & 2 & 3 & 4 & $18 / 60$ & 7 \\
\hline Fondateur & 14 & 16 & 4 & 7 & 5 & $46 / 60$ & 12 \\
Non-fondateur & 4 & 3 & 2 & 2 & 3 & $14 / 60$ & 5 \\
\hline Conseil scientifique & 5 & 3 & 3 & 9 & 6 & $26 / 60$ & 15 \\
Salariés actionnaires & 3 & 3 & 2 & 5 & 4 & $17 / 60$ & 9 \\
Dépôt de brevets & 8 & 4 & 4 & 7 & 7 & $30 / 60$ & 14 \\
Anvar & 11 & 10 & 4 & 6 & 5 & $36 / 60$ & 10 \\
\hline
\end{tabular}

Chaque dirigeant s'est vu attribuer un profil dominant ${ }^{4}$ de formation (scientifique versus gestionnaire). Il est aussi le fondateur ou non de l'entreprise. Respectivement 18 et 14 dirigeants ne sont pas des scientifiques (formation en gestion) ou ne sont pas les fondateurs de l'entreprise. La liaison entre ces deux situations est forte puisque 11 des dirigeants non fondateurs sont de formation gestion (initiale ou par expérience professionnelle). Surtout, ces non-fondateurs, souvent non scientifiques, sont essentiellement présents dans les entreprises à forte croissance potentielle $(4 \mathrm{a}$ et $4 \mathrm{~b})$ et dans une moindre mesure dans les entreprises à stratégie de type 3 (service à la recherche par contrat). On observe une forte présence de conseils scientifiques pour crédibiliser la démarche scientifique des entreprises auprès des financeurs. Cette quête de reconnaissance du plan de croissance de l'entreprise se traduit par le recours à des dépôts de brevets, voire par l'entrée des salariés dans le capital. Sur ces variables qualitatives, les entreprises de type 3 restent assez proches du modèle organisationnel de celles de type $4 \mathrm{a}$ et $4 \mathrm{~b}$.

En revanche, dans les entreprises aux activités dont les potentialités de croissance restent limitées (type 1 et 2, nouveaux produits ou services sur des niches), les dirigeants sont quasiment tous des fondateurs scientifiques. Ils conservent une part du capital avec une majorité de contrôle. Les exceptions relèvent de filiales de groupe dont les dirigeants sont généralement d'anciens cadres de la maison mère. La part des salariés actionnaires demeure relativement faible.

4. Une approche plus détaillée du profil du fondateur a été réalisée par ailleurs (Catherine et Corolleur, 2001). 


\subsection{Stratégies de croissance et contraintes de financement}

Les résultats des statistiques descriptives sur les variables continues sont donnés dans le tableau 2. Pour vérifier la fiabilité d'une approche par les stratégies de croissance, des tests de Kruskal-Wallis ${ }^{5}$ ont été mis en œuvre pour relever les variables qui sont discriminantes par rapport à notre typologie. Les tests d'hypothèse de la normalité des données ont été rejetés avec les moyennes. Dès lors, nous proposons les données sous la forme de médianes qui ont satisfait aux tests de normalité.

TABLEAU 2

Statistiques descriptives sur les variables continues sous forme de médianes (données financières en $\mathrm{K} €$ )

\begin{tabular}{lcccccc}
\hline $\begin{array}{l}\text { Stratégies de croissance } \\
\text { Variables } \\
\text { de management }\end{array}$ & $\begin{array}{c}\mathbf{1} \\
\text { Niche } \\
\text { produit }\end{array}$ & $\begin{array}{c}\mathbf{2} \\
\text { Niche } \\
\text { service }\end{array}$ & $\begin{array}{c}\text { Service } \\
\text { par contrat } \\
\text { recherche }\end{array}$ & $\mathbf{R - D}$ & $\begin{array}{c}\mathbf{4 b} \\
\text { Entrée } \\
\text { marché }\end{array}$ & Total \\
\hline Nombre d'entreprises & 18 & 19 & 6 & 9 & 8 & 60 \\
Création & 1993 & 1991 & 1993 & 1995 & 1987 & 1992 \\
Chiffre d'affaires & 938,6 & 617,1 & 317,5 & 57,3 & 7287,8 & 800 \\
Résultat d'exploitation & $-7,6$ & 15,2 & $-525,5$ & $-1501,6$ & $-1524,5$ & -14 \\
Résultat net & 4,6 & 6,6 & $-491,2$ & $-1178,5$ & $-1933,5$ & $-3,5$ \\
Capital social & 290,1 & 79,3 & 1912,5 & 466,7 & 2441,3 & 258,5 \\
Fonds propres & 500,1 & 117,4 & 2118,7 & 4210,1 & 13852,3 & 541 \\
Effectifs & 8 & 9 & 18 & 19 & 110 & 11 \\
\hline
\end{tabular}

Une première analyse des valeurs médianes ressort du tableau 2. Les entreprises à stratégies de croissance de type 1 et 2 ont des caractéristiques plutôt traditionnelles, avec un chiffre d'affaires et des résultats nets positifs. Les entreprises à stratégie de croissance de type 4a sont plus récentes et ont des chiffres d'affaires très faibles comparées aux entreprises de type $4 \mathrm{~b}$ (mise sur le marché de produits). Les coûts de recherche et développement de ces entreprises (4a et $4 b$ ) sont très élevés, ce qui explique des résultats nets déficitaires. Les entreprises à stratégie de croissance de type 3 semblent être dans une position intermédiaire entre entreprises traditionnelles et entreprises à forte capacité de croissance.

Les contraintes de financement varient profondément selon les stratégies de croissance. Nous avons construit une matrice de corrélation entre les différentes variables financières selon les différents types d'entreprises retenus (tableau 3).

5. Un test non paramétrique est réalisé ; les variables ne suivent pas une loi normale sur la totalité de l'échantillon. 
TABLEAU 3

Matrice de corrélation sur les variables financières

\begin{tabular}{|c|c|c|c|c|c|}
\hline Stratégies de croissance & ce & 2 & 3 & $4 a$ & $4 b$ \\
\hline $\begin{array}{l}\text { Variables } \\
\text { de management }\end{array}$ & $\begin{array}{l}\text { Niche } \\
\text { produit }\end{array}$ & $\begin{array}{l}\text { Niche } \\
\text { service }\end{array}$ & $\begin{array}{l}\text { Service } \\
\text { par contrat } \\
\text { recherche }\end{array}$ & R-D & $\begin{array}{l}\text { Entrée } \\
\text { marché }\end{array}$ \\
\hline Positive & $\begin{array}{l}\text { RE/CA } 0,76^{*} \\
\text { KS/CA } 0,65^{* *} \\
\text { Df/CA } 0,80^{*} \\
\text { DF/KS } 0,6^{* *} \\
\text { FP/RN } 0,9^{*} \\
\text { FP/CA } 0,79^{*} \\
\text { Eff/CA } 0,8^{*}\end{array}$ & $\begin{array}{l}\text { RE/CA } 0,65^{*} \\
\text { FP/KS } 0,87^{*} \\
\text { DF/KS } 0,8^{*} \\
\text { Eff/CA } 0,6^{* *} \\
\text { DF/FP } 0,69^{*}\end{array}$ & & $\begin{array}{l}\mathrm{Liq} / \mathrm{FP} 0,9^{*} \\
\mathrm{Br} / \mathrm{FP} 0,9^{*} \\
\text { Eff/KS 0,8** }\end{array}$ & $\begin{array}{l}\text { DF/CA } 0,8^{* *} \\
\text { FP/KS } 0,8^{*} \\
\text { Liq/augK 0,9* } \\
\text { Brevet/KS 0,8** } \\
\text { Brevet/FP } 0,83^{* *}\end{array}$ \\
\hline Négative & & & & $\mathrm{KS} / \mathrm{RE} 0,85^{*}$ & $\begin{array}{l}\text { FP/RE } 0,85^{*} \\
\text { Liq/RE } 0,89^{*}\end{array}$ \\
\hline
\end{tabular}

$*$ au seuil de $5 \% ; * *$ au seuil de $10 \%$.

Les entreprises développant les stratégies de croissance de type 1 et 2 relèvent d'un modèle économique relativement traditionnel. On note une liaison positive entre : le chiffre d'affaires et le résultat d'exploitation, les dettes financières et le capital social, les fonds propres et le résultat net, les effectifs et le chiffre d'affaires... Leur potentiel de croissance est relativement limité, car le chiffre d'affaires repose en général sur quelques produits ou services standardisés. Ces entreprises présentent des données de comptabilité financière classique du type «soit je fais des profits, soit je meurs à terme».

L'analyse des entreprises à stratégies de croissance $4 a$ ou $4 b$ fournit des résultats plus originaux. Contrairement au contexte général (Mustar, 2001, p. 85), ces entreprises ne s'autofinancent pas et les sources de financement externe ne reposent pas sur l'emprunt, les pouvoirs publics ou la maison mère. Les coûts de recherche-développement sont généralement élevés et nécessitent des financements importants qui passent essentiellement par les marchés financiers à partir d'augmentations de capital. Ces entreprises de type jeunes pousses (stratégies de croissance de type 4a) connaissent un déficit chronique qui se traduit par une corrélation négative entre le capital social et le résultat d'exploitation. Les entreprises à stratégie de croissance de type $4 \mathrm{~b}$ donnent des signaux de plus grande maturité. Bien que les variables financières restent assez atypiques par rapport au modèle économique traditionnel, les corrélations positives entre les dépôts de brevets ou le chiffre d'affaires avec les dettes financières fournissent une information plus en phase avec des critères économiques et financiers traditionnels.

Les entreprises à stratégies de croissance $4 \mathrm{a}$ ou $4 \mathrm{~b}$ n'ont pas vocation à s'autofinancer, ce qui se traduit par une corrélation négative entre le résultat d'exploitation (RE) et le capital social (KS). Cela est d'autant plus vrai que le coût 
de développement d'une molécule qui passe en phase 4, c'est-à-dire la mise sur le marché d'un médicament, nécessite, selon l'entreprise Transgène, un investissement de près de 50 millions d'euros. Devant ces énormes besoins de financement, seuls les fournisseurs du capital-risque et le marché financier peuvent intervenir. Il y a de manière consciente un partage des risques. L'apporteur de capitaux fait un pari sur l'avenir en se basant, dans un premier temps, sur des informations plus qualitatives que financières.

Pour les entreprises à stratégie de croissance de type 3, on relève l'absence de corrélation entre les variables. Plusieurs explications peuvent être avancées : la relative faiblesse de l'échantillon avec six entreprises assez hétérogènes, l'âge des entreprises puisque deux de ces dernières ont moins de trois ans; l'effet Genset (gros chiffre d'affaires, effectifs élevés, pertes élevées, etc.). Il est donc difficile de tirer des enseignements des entreprises à stratégie de croissance de type 3.

\subsection{Synthèse sur les stratégies de comportement des entreprises}

La spécificité des entreprises de biotechnologies (et de la nouvelle économie) remet en question les archétypes distinctifs de l'entreprise entrepreneuriale et de l'entreprise managériale. De nombreux dirigeants-fondateurs se trouvent dans une position hybride entre l'entrepreneur et le manager sous l'effet du développement d'une relation d'agence avec des actionnaires plus ou moins choisis (les fournisseurs du capital-risque).

Dans le domaine de la recherche-développement, les informations sont nettement différentes selon les stratégies de croissance. Dans les entreprises à stratégie de croissance de type $4 \mathrm{a}$ ou $4 \mathrm{~b}$, la valorisation à long terme dépend de la qualité de la recherche engagée (de sa probabilité de succès). Le conseil scientifique, le portefeuille de brevets et/ou de licences et les phases de développement des molécules constituent des signaux de la qualité potentielle de cette recherche. Même si les actionnaires (fournisseurs du capital-risque, public) demandent des informations financières précises, ce sont les besoins de financement séquentiels (financés par augmentation de capital auprès des fournisseurs du capital-risque et/ou du marché financier) pour arriver à développer et à mettre sur le marché une innovation qui vont participer au gouvernement de l'entreprise. Dans ce contexte, il y a passage d'un raisonnement basé sur des états stationnarisés, la valeur d'option dans le cas de la gestion de l'incertitude (Henry, 1974), à la prise en compte d'états turbulents, voire controversés, ce qui pose la question de la création et de l'apprentissage sur de nouveaux états. Ainsi, « cette vision de correction et d'adaptation de l'environnement (création de nouveaux marchés, de nouveaux contextes organisationnels, etc.) par les choix des acteurs (dirigeant, actionnaires, etc.) correspond bien à la vision que peuvent avoir les acteurs investissant dans la nouvelle économie » (Richard et Trommetter, 2001, p. 751). 
Alors que dans les entreprises à stratégies de croissance de type 1 et 2 , le financement des investissements par les banques est généralement retenu, dans celles à stratégies de croissance de types 3, 4a et 4b, un financement par les fournisseurs du capital-risque ou par le marché des capitaux sera privilégié.

Les financements par des acteurs publics (tel l'Anvar) ne semblent pas correspondre à une vision particulière de la stratégie des entreprises puisqu' on les retrouve aussi bien dans les entreprises de type 1 que du type 4 . Néanmoins, les sommes en jeu ne sont pas comparables.

Enfin, on remarquera que les indicateurs de croissance traditionnels ne sont pas opérationnels pour les jeunes pousses de l'industrie des biotechnologies (stratégies de croissance de type $4 \mathrm{a}$ et $4 \mathrm{~b}$ notamment) : les effectifs, le chiffres d'affaires et la valeur ajoutée (pour une vision synthétique complète, voir annexe I). Certaines entreprises dites jeunes pousses ne doivent leur rythme exceptionnel de croissance qu'à la forte progression de leur capital social et / ou de leur capitalisation boursière. À défaut, elles peuvent très bien réaliser peu ou pas de chiffres d'affaires, engendrer pas ou peu de valeur ajoutée (stratégie d'externalisation par la sous-traitance) et compter des effectifs réduits. Quelques données de l'entreprise Nicox illustrent le propos.

TABLEAU 4

Données financières atypiques : l'exemple de Nicox*

\begin{tabular}{lrrrr}
\hline $\begin{array}{l}\text { Données Nicox } \\
\text { en K } €\end{array}$ & $\mathbf{1 9 9 8}$ & $\mathbf{1 9 9 9}$ & $\mathbf{2 0 0 0}$ & $\mathbf{2 0 0 1}$ \\
\hline Chiffre d'affaires & 357 & 227 & 5610 & 0 \\
Résultat d'exploitation & -450 & -4359 & -4547 & -15693 \\
Résultat net & -259 & -3633 & -3008 & -11525 \\
Effectifs & 8 & 22 & 33 & 45 \\
Capital social & 2652 & 2403 & 2421 & 2769 \\
\hline
\end{tabular}

* Les données sur la valeur ajoutée ne sont pas disponibles.

\section{Stratégies de croissance, structures de propriété et contextes de gouvernement d'entreprise}

La structure de propriété est liée aux besoins de financement de l'entreprise euxmêmes consécutifs au choix de la stratégie de croissance. Après vérification de ce point, il est possible de proposer une caractérisation des contextes de gouvernement d'entreprise dans les entreprises de biotechnologie. 


\subsection{Liaison entre les stratégies de croissance et les structures de propriété des entreprises de biotechnologies}

Dans le tableau 5, nous présentons les résultats du croisement entre les variables stratégie de croissance et structure de propriété.

TABLEAU 5

Le croisement des variables stratégies de croissance et structures de propriété

\begin{tabular}{|c|c|c|c|c|c|c|c|}
\hline $\begin{array}{l}\text { Stratégies de croissance } \\
\text { Structures } \\
\text { de propriété }\end{array}$ & $\begin{array}{c}\text { e } 1 \\
\text { Niche } \\
\text { produit }\end{array}$ & $\begin{array}{c}2 \\
\text { Niche } \\
\text { service }\end{array}$ & $\begin{array}{c}3 \\
\text { Service } \\
\text { par contrat } \\
\text { recherche }\end{array}$ & $\begin{array}{c}4 a \\
R-D\end{array}$ & $\begin{array}{c}\text { 4b } \\
\text { Entrée } \\
\text { marché }\end{array}$ & $4 a+b$ & Total \\
\hline 1 & & & & & & & \\
\hline $\begin{array}{l}\text { Entrepreneuriales } \\
2\end{array}$ & 8 & 13 & 1 & 2 & 2 & 4 & 26 \\
\hline Filiales & 4 & 2 & 1 & 0 & 1 & 1 & 8 \\
\hline $\begin{array}{l}3 \mathrm{ab} \\
\text { Capital-risque }<50 \%\end{array}$ & 6 & 4 & 1 & 0 & 1 & 1 & 12 \\
\hline $\begin{array}{l}3 \mathrm{c} \\
\text { Capital-risque }>50 \%\end{array}$ & 0 & 0 & 2 & 6 & 0 & 6 & 8 \\
\hline $\begin{array}{l}4 \\
\text { Présence en Bourse }\end{array}$ & 0 & 0 & 1 & 1 & 4 & 5 & 6 \\
\hline Total & 18 & 19 & 6 & 9 & 8 & 17 & 60 \\
\hline
\end{tabular}

Il est difficile de pratiquer des tests statistiques avec de petits échantillons ou alors il est souvent nécessaire de procéder à des regroupements. Néanmoins, la démonstration est faite de la liaison entre les deux variables (voir annexe II et test du chi carré) mais avec l'inconvénient de perdre de l'information.

Dans le cas présent, hors test statistique, il apparaît clairement que les situations de contrôle majoritaire par les «fournisseurs du capital-risque » (3c) ou l'introduction en Bourse (4) ne se retrouvent pas dans les contextes de stratégies de croissance de type 1 (nouveau produit sur des marchés de niche) ou de type 2 (nouveau service sur des marchés de niche). En revanche, ces structures de propriété $3 \mathrm{c}$ et 4 sont présentes essentiellement dans les activités $4 \mathrm{a}$ et $4 \mathrm{~b}$ (imposant programme de recherche pour des marchés aval de grande dimension, par exemple nouvelle thérapie pour un cancer). Pour les entreprises avec une stratégie de croissance de type 3 (service par contrat de recherche), on retrouve les mêmes conclusions que précédemment pour les données financières. On ne peut reconnaître un modèle de comportement homogène de ces entreprises puisqu'il y a une forte dispersion des structures de propriété. 
Les structures de propriété entrepreneuriale (1) ou de groupe (2) ou avec des fournisseurs du capital-risque possédant moins de $50 \%$ du capital (3ab) se rencontrent dans les entreprises à stratégies de croissance de type 1 ou 2 (marchés de niche de produits ou services). Un approfondissement des cas hybrides met en avant des situations d'entreprise opérant une transition entre deux types de stratégies de croissance. Par exemple, parmi les entreprises à structure de propriété entrepreneuriale, indépendante (1), quatre exercent des activités de recherche de type 4ab, certaines ayant retardé délibérément l'arrivée inéluctable des fournisseurs du capital-risque pour financer l'entreprise.

Il est alors possible de proposer une nouvelle représentation liant les stratégies de croissance rencontrées dans l'industrie des biotechnologies et les acteurs du financement.

\section{FIGURE 1}

\section{Définition des quatre types d'entreprise et du financement associé}

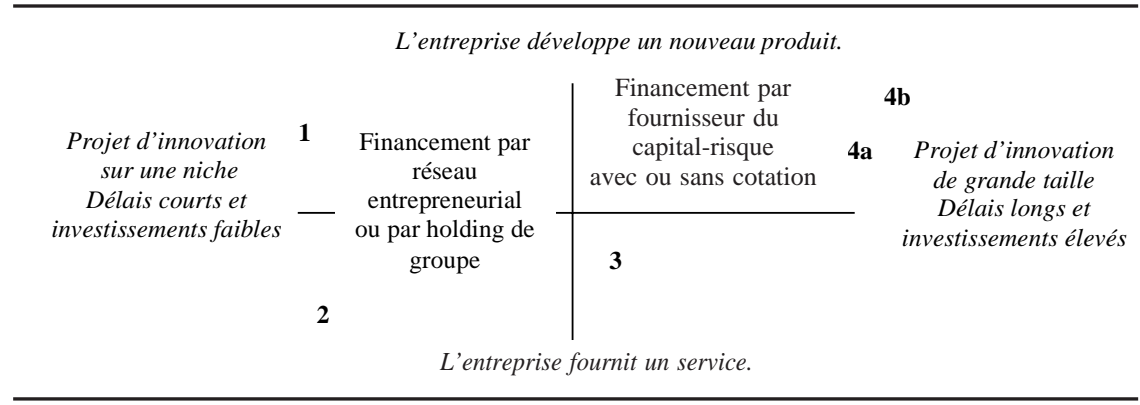

Les quatre types d'activité sont définis autour de deux axes :

- Le premier représente ce que l'on entend par produit. D'un côté, certaines entreprises auront pour objectif de créer un produit dont elles assureront le développement à des fins de commercialisation. De l'autre, certaines entreprises se positionneront plutôt en tant que prestataire de service pour d'autres organisations, en évitant ainsi d'assurer le développement de nouveaux produits. Les innovations portent alors sur les services qui sont offerts.

- Le deuxième axe matérialise les moyens nécessaires au développement de l'innovation. Suivant les cas, celle-ci correspond à un nouveau produit ou à une prestation de service. Les projets de «petite taille» sont associés à un financement lié fréquemment au réseau entrepreneurial du dirigeantfondateur (investisseur providentiel, amorçage et incubateurs, capitalrisque régional) ou à des apports de holding de groupe. En revanche, les 
grands projets de recherche supposent un financement par les fournisseurs du capital-risque. Ils peuvent nécessiter une introduction en Bourse pour lever de nouveaux capitaux ou, pour le moins, rendre plus liquide le capital de la société (permettre les entrées et les sorties des fournisseurs du capital-risque). Ce dernier contexte de financement caractérise celui des « jeunes pousses » : projet de recherche de grande dimension et anticipations crédibles afin, par exemple, de concevoir une thérapie contre des maladies (un marché de grande taille).

Finalement, toutes les stratégies de croissance des entreprises de biotechnologies ne s'inscrivent pas dans le scénario de la jeune pousse. Toutefois, les entreprises de biotechnologies semblent nécessiter plus de capitaux du marché financier que les entreprises à forte croissance, toutes industries confondues. Ainsi, dans notre échantillon, $10 \%$ des entreprises sont introduites en Bourse (structure de propriété 4) et $43 \%$ (structures de propriété 3abc et 4) ont des fournisseurs du capital-risque. Julien (2000) présente des répartitions plus faibles, respectivement $2 \%$ et $12 \%$, dans son échantillon de PME en forte croissance sur différentes industries au Québec.

\subsection{Contextes des mécanismes du gouvernement d'entreprise dans l'industrie des biotechnologies}

Joffre et Wickam (1997) invitent à dépasser les références statistiques et à intégrer des critères qualitatifs pour apprécier la taille, en particulier de la moyenne entreprise. Dans cet esprit, Le Vigoureux (1997) propose une grille d'analyse issue des droits de propriété et comportant deux dimensions principales, l'exclusivité dans l'usage du bien et la transférabilité de la propriété de ce bien. Ainsi, la moyenne entreprise patrimoniale (hors filiales de groupe) occupe une position intermédiaire entre la petite entreprise (forte exclusivité du dirigeant et faible transférabilité) et la grande entreprise (faible exclusivité avec, par exemple, la séparation des droits de contrôle et de gestion et forte transférabilité des droits sur la base d'une cotation en Bourse). La moyenne entreprise est alors structurée avec des droits de direction atténués en associant des cadres à la gestion. La moyenne entreprise n'est pas cotée, ou seulement une part minoritaire du capital, ce qui limite la transférabilité des droits.

L'entreprise des biotechnologies se rapproche largement de ce profil de l'entreprise moyenne. La spécificité de l'industrie, avec la place de la recherche et la prépondérance de dirigeants-fondateurs scientifiques, demande une structuration de l'entreprise et donc une perte d'exclusivité. Même si quelques entreprises sont cotées, leurs transférabilités restent limitées. En effet, la part du capital introduite en Bourse est rarement majoritaire et le poids de certains investisseurs pèserait sur les cours en cas de volumes échangés excessifs. 
En revanche, Le Vigoureux (1997, p. 78 et suivantes) cherche à inférer le comportement stratégique de l'entreprise de la structure de propriété. La structure de propriété serait une variable clé du comportement stratégique. Pour l'heure, sur un plan statistique, nous nous contenterons de souligner la seule liaison entre les deux variables plutôt qu'à expliquer l'une par l'autre. S'il fallait se positionner sur l'influence des variables, nous plaiderions plutôt pour un inversement du modèle. Ainsi, ce serait davantage le comportement stratégique du dirigeant et, plus généralement, de l'entreprise qui serait une variable clé du choix de structure de propriété. Nous rejoignons ainsi davantage le paradigme du management stratégique (Martinet et Reynaud, 2001) en évitant de confondre les buts (le comportement stratégique) et les contraintes (par exemple, le financement et les structures de propriété). Il apparaît clairement, dans cette jeune industrie des biotechnologies, que la structure de propriété évolue en fonction des besoins de financement corrélatifs à la stratégie de croissance. Cette stratégie relève des choix originels de positionnement et d'activité réalisés par l'entrepreneur. Ce n'est pas la structure de propriété qui explique la stratégie de croissance mais davantage le profil de l'entrepreneur et son plan d'affaires. Le comportement de l'entrepreneur des biotechnologies se retrouve largement dans la typologie Croissance - Autonomie - Pérennité versus Pérennité Indépendance - Croissance (Julien et Marchesnay, 1988; Marchesnay, 1998) ${ }^{6}$ : une alternative entre une stratégie de croissance forte et l'ouverture du capital (type 4 et 3c) et une stratégie de croissance limitée par l'autofinancement et l'indépendance du capital (type 1). Dès lors, il n'est plus étonnant ${ }^{7} \mathrm{~d}^{\text {'avoir des entrepre- }}$ neurs innovants, prenant des risques mais adossés à des structures externes (type capital-risque dans les biotechnologies) nécessaires au financement des projets.

Il demeure que la structure de propriété influence la relation d'agence (Charreaux, 1991 ou 1997), donc les pratiques de gouvernement d'entreprise. Charreaux (1997, p. 1) propose la définition suivante d'un système de gouvernement d'entreprise : «Le gouvernement des entreprises recouvre l'ensemble des mécanismes organisationnels qui ont pour effet de délimiter les pouvoirs et d'influencer les décisions des dirigeants, autrement dit, qui "gouvernent" leur conduite et définissent leur espace discrétionnaire. »

Les actionnaires exercent leur pouvoir de contrôle surtout lorsque le dirigeant est minoritaire (structures de propriété $3 c$ et 4) ou qu'il est salarié d'une filiale dans un groupe (structure de propriété 2). Cela explique le souci de dirigeants d'entreprises de biotechnologies reconnaissant dans les entretiens veiller à ne pas perdre le contrôle de l'entreprise «pour éviter la pression du court terme avec les fournisseurs du capital-risque ».

6. L'analyse pourrait être réalisée aussi en termes de légitimité territoriale et légitimité concurrentielle (Marchesnay, 1998).

7. Voir l'étonnement de Le Vigoureux (1997, p. 83) devant ce type de résultats. 


\section{Perspectives}

L'industrie des biotechnologies n'est pas homogène; les stratégies de croissance et les structures de propriété sont diverses. Nous avons relevé quatre types de stratégies de croissance liés à des profils de dirigeants que nous avons confrontés à cinq types de structure de propriété. Le constat est que l'industrie française des biotechnologies trouve les moyens de sa croissance en développant une qualité particulière d'informations malgré un environnement financier annoncé défavorable (Belze et Gauthier, 2000).

Un premier groupe d'entreprises se caractérise par un potentiel de croissance limitée, avec un actionnariat plutôt stable et un financement par les banques. Cet ensemble couvre des filiales de groupes mais surtout des entreprises à structure de propriété indépendante, entrepreneuriale. Parfois, les fournisseurs du capitalrisque arrivent (changement d'activité). Les innovations sont d'abord protégées par le secret. Néanmoins, dans ce même groupe, il existe quelques entreprises dont les perspectives de croissance sont plus fortes. Elles s'appuient sur la présence de fournisseurs du capital-risque dont la part dans le capital reste inférieure à $50 \%$. La présence de ces investisseurs modifie les mécanismes de gouvernement d'entreprise qui demeurent proches de ceux d'une petite entreprise avec une place importante laissée au rôle de la comptabilité financière. Les banques jouent un rôle prépondérant dans le financement. La référence aux concepts de jeune pousse et de nouvelle économie revêt un caractère quelque peu usurpé pour caractériser ces entreprises et leur contexte environnemental.

En revanche, le second groupe est lié à des entreprises à fort potentiel de croissance, les fameuses jeunes pousses ou gazelles (Julien, 2001, citant Birch, Haggerty et Parsons, 1997). Ce sont des entreprises de recherche qui vont passer au stade de développement et de commercialisation à un horizon de 10 à 15 ans, selon les activités. Les mécanismes de gouvernement d'entreprise seront fortement liés aux nécessaires augmentations de capital finançant les programmes de recherche par phase de trois ans. L'information de la comptabilité financière n'apporte que peu d'éléments tangibles pour la prise de décision de l'apporteur de capitaux, essentiellement l'actionnaire. Celui-ci sera plus sensible à des éléments scientifiques (dépôts de brevets, composition du conseil scientifique), signaux déjà soulignés par Julien (2001) pour les entreprises à forte croissance. Ce contexte environnemental peut être qualifié de nouvelle économie. Les indicateurs statistiques traditionnels (effectifs, chiffre d'affaires, valeur ajoutée) sont insuffisants pour caractériser la taille des entreprises. Les nombreuses entreprises de biotechnologies, sans chiffre d'affaires et avec moins de 50 salariés, relèvent de la moyenne entreprise au regard d'un processus de séparation des droits de contrôle et de gestion et d'une ouverture du capital favorisant la transférabilité des droits. 
Les structures de propriété ne sont qu'un des mécanismes organisationnels susceptibles d'intervenir. L'influence des autres mécanismes de gouvernement d'entreprise issus de la seule relation du dirigeant avec les actionnaires ou du dirigeant avec les autres partenaires de l'entreprise (Charreaux, 1997; Boissin, 2000a et b; Boissin, Guieu et Wirtz, 2001 ; Martinet et Reynaud, 2001) n'est pas intégrée dans ce travail.

\section{ANNEXE I}

Tableau de synthèse

\begin{tabular}{|c|c|c|c|c|c|}
\hline Typologie (1) & 1 & 2 & 3 & $4 a$ & $4 b$ \\
\hline $\begin{array}{l}\text { Variables } \\
\text { de management }\end{array}$ & $\begin{array}{c}\text { Niche } \\
\text { produit }\end{array}$ & $\begin{array}{l}\text { Niche } \\
\text { service }\end{array}$ & $\begin{array}{l}\text { Service } \\
\text { par contrat } \\
\text { recherche }\end{array}$ & R-D & $\begin{array}{l}\text { Entrée } \\
\text { marché }\end{array}$ \\
\hline Chiffre d'affaires & $=$ & -- & --- & ---- & ++ \\
\hline Résultat d'exploitation & $=$ & $=$ & -- & ---- & --- \\
\hline Résultat net & $=$ & $=$ & -- & --- & -- \\
\hline Dette financière & $=$ & -- & & & \\
\hline Dirigeant scientifique & $=$ & $=$ & - & --- & -- \\
\hline Dirigeant-fondateur & $=$ & $=$ & - & --- & --- \\
\hline Conseil scientifique & $=$ & $=$ & + & ++ & ++ \\
\hline Brevets & $=$ & -- & + & ++ & ++ \\
\hline $\begin{array}{l}\text { Phase de développement } \\
\text { des molécules }\end{array}$ & $=$ & $=$ & $=$ & + & ++ \\
\hline Anvar & $=$ & $=$ & $=$ & $=$ & $=$ \\
\hline
\end{tabular}

\section{ANNEXE II}

\section{Regroupement de catégories et liaison entre les variables Activité et Structure de l'actionnariat}

\begin{tabular}{lccccc}
\hline $\begin{array}{l}\text { Stratégies de croissance } \\
\begin{array}{l}\text { Structures } \\
\text { de propriété }\end{array}\end{array}$ & $\begin{array}{c}\mathbf{1} \\
\text { Niche } \\
\text { produit }\end{array}$ & $\begin{array}{c}\text { Niche } \\
\text { service }\end{array}$ & $\begin{array}{c}\text { Service } \\
\text { par contrat } \\
\text { recherche }\end{array}$ & $\begin{array}{c}\text { 4ab } \\
\text { Recherche } \\
\text { et entrée } \\
\text { marché }\end{array}$ & Total \\
\hline let 2 & $12(10,2)$ & $15(10,8)$ & $2(3,4)$ & $5(9,6)$ & 34 \\
\hline 3abc et 4 & $6(7,8)$ & $4(8,2)$ & $4(2,6)$ & $12(7,4)$ & 26 \\
\hline Total & 18 & 19 & 6 & 17 & 60
\end{tabular}

Les effectifs théoriques sont donnés entre parenthèses. La valeur du chi carré est de 11. Il existe un lien entre les deux typologies retenues. 


\section{Bibliographie}

AudRETSCH, D.B. et P.E. STEPHAN (1996), «Company-scientist locational links : the case of biotechnology », American Economic Review, vol. 86, nº 3, p. 641-652.

BELZE, L. et O. GAUTHIER (2000), « Innovation et croissance économique : rôle et enjeux du financement des PME», Revue internationale PME, vol. 13, n 1, p. 65-86.

Birch, D., A. Haggerty et W. PARsons (1997), Corporate Almanac, Cambridge, Cognetic Inc.

BoIssin, J.P. (2000a), «Le groupe et ses acteurs dans leur environnement économique et social», dans J. Richard (dir.), Analyse financière et gestion des groupes, Paris, Economica, chapitre 20, p. 381-403.

BoIssin, J.P. (2000b), «La vulnérabilité de la théorie normative de l'agence : l'exemple des relations actionnaires-dirigeants d'un holding familial», dans Vulnérabilité et droit - Le développement de la vulnérabilité et ses enjeux en droit, Grenoble, Presses universitaires de Grenoble, p. 101-116.

BOISSIN, J.P., G. GUIEU et P. WIRTZ (2001), «Les fusions internationales - une lecture à travers la théorie institutionnelle», dans A.C. Martinet et R.A. Thiétard (dir.), Stratégies : actualités et futurs de la recherche, Paris, Vuibert, chapitre 16, p. 249-272.

Castagnos, J.C., J.P. Boissin et G. Guieu (1997), «Revues francophones et recherche en stratégie», Économies et Sociétés, série Sciences de Gestion, nº 23, p. 37-73.

CATHerine, D. et F. Corolleur (2001), «Nouvelles entreprises de biotechnologies et géographie de l'innovation, des fondateurs à leur modèle d'entreprise », Revue d'économie régionale et urbaine, $\mathrm{n}^{\circ}$ 5, p. 785-808.

CharreauX, G. (1991), «Structure de propriété, relation d'agence et performance financière », Revue économique, vol. $42, \mathrm{n}^{\circ} 3$, mai, p. 521-552.

CharreauX, G. (1997), «Introduction générale et vers une théorie du gouvernement d'entreprise», dans G. Charreaux (dir.) Le gouvernement des entreprises : théories et faits, Paris, Economica, p. 1-13 et p. 421-469.

HENRY, C. (1974), «Investment decision under uncertainty : the irreversibility effect», American Economic Review, vol. 64, p. 1006-1012.

HuBERMAN, A.M. (1981), « Splendeurs, misères et promesses de la recherche qualitative », Éducation et recherche, vol. 3, p. 233-249.

Huberman, A.M. et M.B. Miles (1991), Analyses de données qualitatives : Recueil de nouvelles méthodes, Paris, De Boeck Université, 480 p.

JOFFRE, P. et S. WICKAM (1997), «Les atouts des entreprises moyennes », Revue française de gestion, $\mathrm{n}^{\circ} 116$, novembre-décembre, p. 64-70.

Joly, P.B., S. Lemarié, et V. Mangematin (1998), «Coordination et incitations dans les contrats de recherche», Revue économique, $\mathrm{n}^{\circ}$ 49, p. 1129-1147.

JULIEN, P.-A. (2000), «Les PME à forte croissance : les facteurs explicatifs», Actes de la $I X^{e}$ conférence de l'Association internationale de management stratégique, CD-ROM, Montpellier, 24-26 mai. 
JuliEN, P.-A. (2001), «Les PME à forte croissance et la métaphore du jazz: Comment gérer l'improvisation de façon cohérente», Revue internationale PME, vol. 14, $\mathrm{n}^{\text {os }} 3-4$, p. 129-161.

Julien, P.-A. et M. MARCheSnAy (1988), La petite entreprise, Paris, Vuibert.

LE VigOUREUX, F. (1997), «Entreprises moyennes : structures de propriété et comportement stratégique », Revue française de gestion, $\mathrm{n}^{\circ}$ 116, novembre-décembre, p. 71-84.

Mangematin, V., S. Lemarié, J.-P. Boissin, D. CATherine, F. Corolleur, R. Coronini et M. TROMmETTER (2003), «Development of SMEs and heterogeneity of trajectories : the case of biotechnology in France », Research Policy, vol. 32, n 4, p. 621-638.

MARChESNAY, M. (1998), «Confiances et logiques entrepreneuriales », Économies et Sociétés, série Sciences de Gestion, numéro spécial $20^{\mathrm{e}}$ anniversaire, $\mathrm{n}^{\text {os }} 8-9$, p. 99-117.

MARTinet, A.C. (1990), «Grandes questions épistémologiques et sciences de gestion: Épistémologie de la stratégie », dans A.C. Martinet (dir.), Épistémologies et sciences de gestion, Paris, Economica, p. 9-29 et p. 211-236.

MARTINET, A.C. (1992), «La recherche en stratégie : une industrie vulnérable ?», Cahier de recherche de l'IAE de Lyon, $\mathrm{n}^{\circ} 10,6 \mathrm{p}$.

MARTinet, A.C. (1997), «Pensée, stratégie et rationalités : un examen épistémologique », Management international, vol. 2, $\mathrm{n}^{\circ} 1$, p. 67-75.

MARTINET, A.C. et E. REYNAUD (2001), « Shareholders, stakeholders et stratégie », Revue française de gestion, $\mathrm{n}^{\circ} 136$, novembre-décembre, p. 12-25.

Mignot, J.P. et C. PONCET (2001), «The industrialisation of knowledge in life science : convergence between public research and industrial strategies », Cahier de recherche du Creden, $\mathrm{n}^{\circ}$ 01.01.20, Montpellier, 17 pages.

MounOUD, E. (1997), L'inscription sociale des discours et des représentations stratégiques dans l'industrie de l'environnement, Thèse du doctorat HEC, Paris.

Mustar, P. (2001), «Diversité et unité des entreprises à forte croissance du secteur manufacturier en France », Revue internationale PME, vol. 14, nos 3-4, p. 67-89.

Richard A. et M. TROMMETTER (2001), «Les caractéristiques d'une décision: effet irréversibilité et endogénéisation de l'environnement », Revue économique, vol. 52, no 3, p. 739-752.

Revue internationale P.M.E., vol. 16, nos 3-4, 2003

(C) 2004 - Presses de l'Université du Québec

Édifice Le Delta I, 2875, boul. Laurier, bureau 450, Sainte-Foy, Québec G1V 2M2 • Tél. : (418) 657-4399 - www.puq.ca

Tiré de : Revue internationale P.M.E., vol. 16, $\mathrm{n}^{\text {os }} 3$-4, sous la direction de Pierre-André Julien • PME1603N Tous droits de reproduction, de traduction et d'adaptation réservés 